Z. Epileptol. $2021 \cdot 34: 306-310$

https://doi.org/10.1007/s10309-021-00427-y

Angenommen: 9. Juni 2021

Online publiziert: 7. Juli 2021

(c) Springer Medizin Verlag GmbH, ein Teil von Springer Nature 2021

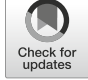

Randi von Wrede $\cdot$ Rainer Surges

Klinik und Poliklinik für Epileptologie, Universitätsklinikum Bonn, Bonn, Deutschland

\title{
Patient-zu-Arzt-Anwendungen in der ambulanten Epilepsieversorgung
}

behandlungsverbot wurde 2018 von der Bundesärztekammer aufgehoben. Zwar soll die ärztliche Beratung und Behandlung im persönlichen Kontakt mit den Patient*innen erfolgen, was als Goldstandard gewertet wurde, eine ausschließliche telemedizinische Beratung oder Behandlung sei jedoch im Einzelfall erlaubt, wenn dies ärztlich vertretbar sei, die erforderliche ärztliche Sorgfalt gewahrt bliebe und der Behandelte über die Besonderheiten der ausschließlichen telemedizinische Beratung und Behandlung aufgeklärt würde [11].

\section{Vor- und Nachteile telemedizi- nischer Dienstleistungen} strukturierter telemedizinischer Ansätze $[10,19,25]$. Die COVID-19-Pandemie erforderte, um das Ansteckungsrisiko $\mathrm{zu}$ minimieren und die Ressourcen $\mathrm{zu}$ allokieren, eine schnellere Implementierung telemedizinischer Angebote, um die ambulante medizinische Betreuung insbesondere für chronisch kranke Patient ${ }^{*}$ innen aufrechtzuerhalten und Versorgungsengpässe und Therapieabbrüche zu vermeiden.

\section{Historisch-rechtliche Aspekte}

Das aus dem Geschlechtskrankheitengesetz von 1927 [16] übernommene Fern-

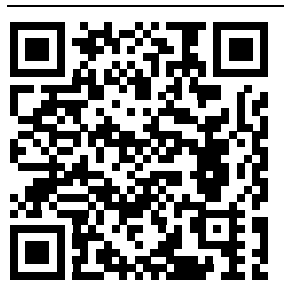

QR-Code scannen \& Beitrag online lesen
Die telemedizinische Behandlung dient als Teil der Telematik im Gesundheitswesen der Diagnostik und Therapie unter Überbrückung einer räumlichen oder auch zeitlichen („asynchron“) Distanz zwischen Patient ${ }^{*}$ in und Arzt/Ärztin [12]. Eine telemedizinische Beratung reduziert den Zeit- und auch Kostenaufwand für den/die Patient*in. Insbesondere Patient*innen mit Behinderungen, ihren Familien und Betreuern erleichtert eine telemedizinische Beratung den Zugang zur fachärztlichen Versorgung. Eine telemedizinische Beratung bietet der/dem Patient*in die Möglichkeit der medizinischen Behandlung in einem sicheren, bequemen, bekannten und geschützten Rahmen. In einem anderen Kontext, Morbus Parkinson, werden diese Vorteile plakativ mit den vier C („better access to care, convenience, comfort, confidentiality") und seit der COVID19-Pandemie mit einem zusätzlichen $C$ („,contagion“) zusammengefasst [3, 4].
Telemedizinische Behandlungen gehen mit reduzierten Kostenaufwendungen für die Patient*innen [6] und Kostenträger (direkte und indirekte Krankheitskosten) einher. Seitens der Leistungserbringer sind Zeiteinsparungen, optimierte Nutzung der Infrastruktur und Qualitätsaspekte wünschenswerte Folgen der Implementierung von telemedizinischen Behandlungen.

Nicht zu unterschätzen sind jedoch auch mögliche Nachteile einer telemedizinischen Behandlung. Historisch fußt die therapeutische Beziehung auf einem persönlichen Kontakt vor Ort. Die gewohnte interpersonelle Interaktion dient dem Aufbau einer vertrauensvollen und nachhaltigen Patient-ArztBeziehung. Neben visuellen und auditiven Kommunikationswegen können hier alle Sinne verwendet werden. Die nichtsprachliche Kommunikation ist bei vielen Patient ${ }^{*}$ innen ein wesentlicher Bestandteil der Behandlung. Patient ${ }^{*}$ innen mit eingeschränktem Seh- oder Hörvermögen fehlen insbesondere diese Wahrnehmungen, die sie zur Ergänzung oder Substitution ihrer Defizite benötigen. Kulturell bedingt, ist die körperliche Berührung ein Vertrauen bildendes Moment der Patienten-Arzt-Beziehung [9, 24], sei es durch den bis zur COVID19-Pandemie üblichen Begrüßungsund Verabschiedungshandschlag, der Anfang und Ende der Interaktion bekräftigte, sei es durch die körperliche Untersuchung. Die Nutzung telemedizinischer Behandlungsoptionen fordert daher eine Neuorientierung hinsichtlich Vertrauen bildender Maßnahmen und eine bewusste und optimierte Nutzung der möglichen visuellen und sprachli- 


\section{Tab. 1 Telemedizin-Etikette}

\section{Patient*in Arzt/Ärztin}

Technik Stabile Internetverbindung

Kameraeinstellung auf Gesicht und oberen Schulterbereich (Position so adjustieren dass Augenkontakt auf Augenhöhe)

Mikrofon mit ausreichender Übertragungsqualität

Umfeld

Ausreichende Lichtverhältnisse

Ruhige Umgebung, die vertrauliche und konzentrierte Behandlung ermöglicht

Kennzeichnung des Raumes, um Unterbrechungen zu vermeiden

Ausschalten nicht genutzter Mobilgeräte (WLAN-Kapazitäten, Ablenkung)

Benennen, wer an der Beratung teilnimmt, Authentifizierung

Benennen, ob Aufnahmen der Beratung zulässig/unzulässig

- $\quad$ Professioneller Hintergrund (am besten gewohnte Praxisumgebung

o. Ä.)

- $\quad$ Professionelle Kleidung

Verhalten/ Pünktlichkeit

Bewegung Respektvolles Verhalten wie im gewohnten ambulanten Kontakt

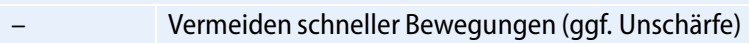

- $\quad$ Terminiertes Ende der Beratung (ggf. vorher ansprechen)

Kommuni- - Klare Kommunikation

kation _ _ Vermeiden schnellen und undeutlichen Sprechens

- $\quad$ Einfache Syntax

- $\quad$ Vermeiden von Fachtermini

- $\quad$ Vergewissern, ob Inhalte verstanden wurden (Wiederholung, Zusammenfassung)

Qualitäts- Wahrnehmung als wirkliche Beratung und Behandlung - keine „Behandlung-to-go" voraussetzung
Vorbereitung wie bei Beratung und Behandlung vor Ort - kein „Drive-in“

Konzentration und Fokussierung wie bei Beratung und Behandlung vor Ort - „kein Zappen"

Nachbereitung wie bei Beratung und Behandlung vor Ort

Vergewissern, ob telemedizinische Behandlung ausreichend war, um Befunderhebung, Beratung durchzuführen, sonst Besuch vor Ort veranlassen chen Kommunikationswege, um fehlende Kommunikationsstrukturen (nicht sprachlich, insbesondere Körpersprache) auszugleichen (s. - Tab. 1). Das Wahrnehmen der Technik-immanenten Unterschiede sowie die Bereitschaft, ein gleiches Maß an Zeit, Aufmerksamkeit, Konzentration und Wertschätzung im telemedizinischen Kontakt zu geben, sind Voraussetzung einer sinnvollen und qualitativ gleichwertigen Behandlung.

Die Implementierung telemedizinischer Ansätze bei chronisch neurologischen Erkrankungen, insbesondere in der Versorgung von Patient ${ }^{\star}$ innen mit Epilepsie war bis zum Ausbruch der COVID-19-Pandemie eher zögerlich. Erste wissenschaftliche Erkenntnisse zur Qualität von telemedizinischer Behandlung von Patient*innen mit Epilepsie aus der Vor-COVID-19-Pandemiezeit stammen, wie zu erwarten, aus Ländern mit großen Entfernungen oder schwierigem bzw. reduziertem Zugang zum Gesundheitssystem [1, 21, 23]. In nie geahnter Geschwindigkeit katalysierte die COVID-19-Pandemie die weltweite Versorgungslandschaft. Ein wesentlicher Baustein war die Reorganisation der ambulanten Versorgungsstrukturen mit Implementierung telemedizinischer Angebote [22].

\section{Technische und administrative Voraussetzungen}

Eine gute und sicher funktionierende Hard- und Software ist für eine hochwertige telemedizinische Beratung unerlässlich. Die Anforderungen hierzu sind in der Anlage 31b zum Bundesmantelvertrag-Ärzte geregelt [13]. Ein Großteil der zu beachtenden gesetzlichen Regelungen ist damit erfüllt. Zertifizierte Videodienstanbieter können unter [14] abgerufen werden. Die Finanzierung der Videosprechstunde ist seit Oktober 2019 klarer geregelt und beinhaltet u. a. eine Grundoder Versichertenpauschale, Zuschläge für die fachärztliche Grundversorgung und für die Authentifizierung eines/ einer unbekannten Patient ${ }^{*}$ in, Anschubfinanzierung und Technikzuschlag. Bei einem zusätzlichen persönlichen Kontakt im Quartal erfolgt die vollständige Vergütung, bei ausschließlicher telemedizinischer Behandlung erfolgt ein Abschlag (20\% bei Neurologen/Kinderund Jugendärzten). Der Anteil der telemedizinischen Sprechstunde ist gedeckelt, diese Einschränkung ist aktuell jedoch noch ausgesetzt [15].

Die Aufklärung des/der Patient*in über die Besonderheiten einer telemedizinischen Beratung ebenso wie die gesamte Beratung sind zu dokumentieren. Die Sorgfalts- und Aufklärungspflicht gilt selbstverständlich im gleichen Umfang, hierzu zählt auch die Versicherung, dass das ausgewählte Medium ausreichend ist, um eine Befunderhebung, Befundkommunikation und Aufklärung durchzuführen. Haftungsrechtlich ist letztendlich noch unklar, wie die erforderliche Sorgfaltspflicht gewertet wird, das beinhaltet selbstverständlich auch Aufklärungen über neu eingeleitete $\mathrm{Be}$ handlungen.

\section{Erfahrungen der Implemen- tierung der Telemedizin in der ambulanten Patienten- versorgung}

Zwischenzeitlich stehen weltweite Erfahrungen mit telemedizinischen Behandlungen von Patient ${ }^{\star}$ innen mit Epilepsie zur Verfügung [5, 7, 8, 17, 20, 26, 27]. Die Mehrzahl der Untersuchungen bezieht sich auf die Ergebnisse der telemedizinischen Sprechstunden während der ersten Corona-Welle im Frühjahr 2020. In Deutschland gelang die Implementierung von telemedizinischen Beratung insgesamt rasch, schon im Sommer 2020 veröffentlichten 2 Schwerpunktkliniken 
ihre Erfahrungen [26, 27]. Insgesamt sind die Akzeptanz und Zufriedenheit von Patient ${ }^{*}$ innen und Behandler*innen groß (bis zu 95\%), die Qualität der telemedizinischen Beratung wird als gleichwertig eingeschätzt, und auch die mögliche Adhärenz ist als ebenwertig zu erwarten. Die vorliegenden Daten wurden jedoch im Wesentlichen unter dem Eindruck und der Änderungsbereitschaft der ersten Corona-Welle erhoben. Erste Hinweise ergaben sich, dass die telemedizinischen Kontakte eher als Zusatzangebot denn als Ersatzangebot aufgefasst wurden. Die Bereitschaft einer weiteren telemedizinischen Beratung war höher bei jüngeren Patient*innen, bei Patient ${ }^{*}$ innen, welche nicht Muttersprachler waren, und solchen, die noch nicht lange am Zentrum angebunden waren. Letzteres reflektiert möglicherweise den Einfluss der persönlichen ArztPatient ${ }^{*}$ in-Bindung [27]. Eine spanische Untersuchung zeigte bei insgesamt hoher Zufriedenheit mit der telemedizinischen Beratung (84\%), dass, wenn auch nicht signifikant, Patient ${ }^{*}$ innen mit Pharmakoresistenz eher einen Besuch vor Ort als eine telemedizinische Behandlung wünschen. Eine positive Korrelation mit telemedizinischer Beratung konnte für Patient ${ }^{*}$ innen mit größeren Ängsten vor COVID-19 gefunden werden [8]. Eine weitere Studie, welche jedoch später in der COVID-19-Pandemie (Juli bis Dezember 2020) durchgeführt wurde, zeigte weiterhin eine hohe Zufriedenheit (74\%) und Vergleichbarkeit mit VorOrt-Besuchen [10]. Eine irische Untersuchung, die die Erfahrungen von Klinikern und Patient ${ }^{\star}$ innen $\mathrm{zu}$ telemedizinischen Behandlungen in einer Prä-Corona-Pandemie-Phase mit denen in der Pandemie vergleicht, zeigte eine hohe Zufriedenheit der Behandler sowie der Behandelten. Bedenken seitens der Behandler wurden für neue Patient ${ }^{*}$ innen, für Patient ${ }^{*}$ innen mit einer unklaren Anfallskontrolle sowie für Epilepsiechirurgiekandidaten geäußert, seitens der Patient ${ }^{\star}$ innen zeigte sich ein höherer Wunsch einer Behandlung im persönlichen Kontakt, wenn sich ihr Gesundheitszustand verschlechtern würde [2].

Z. Epileptol. 2021 · 34:306-310 https://doi.org/10.1007/s10309-021-00427-y

(c) Springer Medizin Verlag GmbH, ein Teil von Springer Nature 2021

\section{R. von Wrede $\cdot R$. Surges}

\section{Patient-zu-Arzt-Anwendungen in der ambulanten Epilepsieversorgung}

\section{Zusammenfassung}

Die telemedizinische Behandlung von Patient*innen mit Epilepsie ist eine Erweiterung der bislang die ambulante Versorgungslandschaft im Wesentlichen prägenden klassischen persönlichen Arztbesuche. Unter administrativen, finanziellen und rechtlichen nun klareren Rahmenbedingungen konnten, durch die COVID-19-Pandemie katalysiert, rasch die zuvor langsameren Prozesse in der Umsetzung beschleunigt werden. Nationale und internationale Erfahrungen verweisen auf eine Noninferiorität im

\begin{abstract}
Vergleich zu Vor-Ort-Besuchen. Verbindliche Algorithmen der Zuordnung Vor-Ort-Besuch vs. telemedizinische Behandlung sind noch ausstehend und sollten zukünftig unter Berücksichtigung der Patientencharakteristika sowie Behandlungssituation erarbeitet werden.
\end{abstract}

\section{Schlüsselwörter}

Telemedizin · Ambulante Behandlung · Videosprechstunde $\cdot$ Qualität · Etikette

\section{Patient-to-doc applications in outpatient epilepsy treatment}

\section{Abstract}

The telemedical treatment of patients with epilepsy is an extension of the classical face to face on-site appointment with treating physicians, which has essentially characterized the outpatient care to date. Under the recently clarified administrative, financial and legal conditions, especially catalyzed by the COVID19 pandemic, the previously slower process in the implementation could be rapidly accelerated. National and international experiences indicate noninferiority of telemedical counseling as compared to onsite appointments. Valid algorithms for the allocation of on-site appointments versus telemedical treatment are still lacking and should be developed in the near future, taking patient characteristics and treatment situations into account.

\section{Keywords}

Telemedicine · Outpatient treatment · Video consultation · Quality · Etiquette

\section{Zusammenfassung und Fazit für die Praxis}

Telemedizinische Behandlungen sind ein sinnvolles und erwünschtes Angebot in der Versorgungslandschaft für Patient*innen mit Epilepsie. Die rechtlichen und finanziellen Rahmenbedingungen wurden mittlerweile klarer definiert, wenn auch haftungsrechtlich noch offene Fragen bestehen. Um den Effekt der Corona-Pandemie auf die Bereitschaft und Zufriedenheit von telemedizinischen Behandlungen zu erfassen, sind langfristig angelegte Projekte notwendig, um die generelle Einschätzung von Behandlern und Behandelten zu erfassen. Eine Herausforderung wird sein, die geeignete Behandlungssituation sowie den/die geeignete/n (und natürlich ungeeignete/n) Patient*in für telemedizinische Behandlungen $\mathrm{zu}$ benennen. Ein interessanter
Ersatz für die Zuordnung von Vor-Ortund telemedizinischen Behandlungen findet sich bei [18], eine Adaptation für Post-Corona-Zeiten ist noch ausstehend. Die - Tab. 2 legt einen Überblick über eine potenzielle Allokation vor, deren Wertigkeit jedoch noch in entsprechenden Versorgungsforschungsprojekten verifiziert oder falsifiziert werden muss.

Die traditionelle Epilepsieversorgung bestand aus einer episodischen, meist reaktiven Vor-Ort-Versorgung im persönlichen Kontakt [2], die bislang vorgelegten Untersuchungen zur telemedizinischen Beratung belegen eine zumindest Nichtunterlegenheit $\mathrm{zu}$ Besuchen vor Ort. Die Implementierung von digitalen Ansätzen in der Gesundheitsversorgung folgt damit den Veränderungen, die andere Bereiche des Dienstleistungssektors bereits durchlaufen sind. Der Besonderheit der Gesundheitsversorgung, insbe- 
Tab. 2 Telemedizin - Vorteile und Nachteile in bestimmten Behandlungssituationen

\begin{tabular}{|c|c|c|c|}
\hline \multicolumn{2}{|c|}{ Behandlungssituation } & \multirow{2}{*}{$\begin{array}{l}\text { Vorteile } \\
\text { Technikaffinität }\end{array}$} & \multirow{2}{*}{$\begin{array}{l}\text { Nachteile } \\
\text { Eingeschränkte körperliche Untersuchung }\end{array}$} \\
\hline Patienten- & Patient*innen $<30$ Jahre & & \\
\hline charakte- & Patient*innen > 60 Jahre & Unabhängig von Mobilität & Eingeschränkte körperliche Untersuchung \\
\hline & Patient*innen mit Behinderungen - körperlich & Unabhängig von Mobilität & Eingeschränkte körperliche Untersuchung \\
\hline & Patient*innen mit Behinderungen - geistig & $\begin{array}{l}\text { Unabhängig von Mobilität; geringerer } \\
\text { Zeitaufwand für Betreuungsperson }\end{array}$ & Eingeschränkte körperliche Untersuchung \\
\hline & Patient*innen mit Sprachbarriere & Dolmetscher ggf. leichter zu organisieren & Eingeschränkte körperliche Untersuchung \\
\hline & $\begin{array}{l}\text { Berufstätige Patient*innen } \\
\text { Patient*innen, die selbst Betreuungsperson sind }\end{array}$ & Geringerer Zeitaufwand & Eingeschränkte körperliche Untersuchung \\
\hline & Patient*innen im Erstkontakt & $\begin{array}{l}\text { Ggf. rasche Einschätzung, welche Fach- } \\
\text { richtung und Behandlungsform ange- } \\
\text { messen }\end{array}$ & Eingeschränkte körperliche Untersuchung \\
\hline \multirow{3}{*}{$\begin{array}{l}\text { Komorbi- } \\
\text { ditäten }\end{array}$} & Komorbiditäten, die die Epilepsie beeinflussen & Schnellerer Zugriff auf Fachexpertise & Eingeschränkte körperliche Untersuchung \\
\hline & $\begin{array}{l}\text { Komorbiditäten, die von der Epilepsie beeinflusst } \\
\text { werden }\end{array}$ & Schnellerer Zugriff auf Fachexpertise & $\begin{array}{l}\text { Eingeschränkte körperliche Untersuchung } \\
\text { Fehlende Zusatzuntersuchungen }\end{array}$ \\
\hline & Neu aufgetretene Komorbiditäten & $\begin{array}{l}\text { Ggf. rasche Einschätzung, welche Fach- } \\
\text { richtung und Behandlungsform ange- } \\
\text { messen }\end{array}$ & $\begin{array}{l}\text { Eingeschränkte körperliche Untersuchung } \\
\text { Fehlende Zusatzuntersuchungen }\end{array}$ \\
\hline \multirow[t]{3}{*}{$\begin{array}{l}\text { Anfalls- } \\
\text { kontrolle }\end{array}$} & Gebessert & $\begin{array}{l}\text { Geringerer Zeitaufwand } \\
\text { Geringerer finanzieller Aufwand }\end{array}$ & - \\
\hline & Unverändert & $\begin{array}{l}\text { Geringerer Zeitaufwand } \\
\text { Geringerer finanzieller Aufwand }\end{array}$ & Fehlende Zusatzuntersuchungen \\
\hline & Verschlechtert & Rasche, zeitnahe Therapieberatung & $\begin{array}{l}\text { Eingeschränkte körperliche Untersuchung } \\
\text { Fehlende Zusatzuntersuchungen }\end{array}$ \\
\hline \multirow{3}{*}{$\begin{array}{l}\text { Neben- } \\
\text { wirkungs- } \\
\text { situation }\end{array}$} & Gebessert & $\begin{array}{l}\text { Geringerer Zeitaufwand } \\
\text { Geringerer finanzieller Aufwand }\end{array}$ & Eingeschränkte körperliche Untersuchung \\
\hline & Unverändert & $\begin{array}{l}\text { Geringerer Zeitaufwand } \\
\text { Geringerer finanzieller Aufwand }\end{array}$ & $\begin{array}{l}\text { Eingeschränkte körperliche Untersuchung } \\
\text { Fehlende Zusatzuntersuchungen }\end{array}$ \\
\hline & Verschlechtert & Rasche, zeitnahe Therapieberatung & $\begin{array}{l}\text { Eingeschränkte körperliche Untersuchung } \\
\text { Fehlende Zusatzuntersuchungen }\end{array}$ \\
\hline \multirow{6}{*}{$\begin{array}{l}\text { Spezielle } \\
\text { Frage- } \\
\text { stellungen }\end{array}$} & Verhütung & - & Aufklärungsumfang, Verständnis \\
\hline & Schwangerschaft & - & Fehlende Zusatzuntersuchungen \\
\hline & Fahrtauglichkeit & - & $\begin{array}{l}\text { Eingeschränkte körperliche Untersuchung } \\
\text { Fehlende Zusatzuntersuchungen } \\
\text { Justiziabilität? }\end{array}$ \\
\hline & Arbeitsmedizinische Fragestellungen & $\begin{array}{l}\text { Screening, Einleitung formaler Beantra- } \\
\text { gungen }\end{array}$ & $\begin{array}{l}\text { Eingeschränkte körperliche Untersuchung } \\
\text { Fehlende Zusatzuntersuchungen } \\
\text { Justiziabilität? }\end{array}$ \\
\hline & Epilepsiechirurgische Fragestellungen & $\begin{array}{l}\text { Ggf. rasche Einschätzung, welche Be- } \\
\text { handlungsform prinzipiell möglich }\end{array}$ & $\begin{array}{l}\text { Eingeschränkte körperliche Untersuchung } \\
\text { Fehlende Zusatzuntersuchungen } \\
\text { Justiziabilität (Aufklärung)? }\end{array}$ \\
\hline & Stimulationsverfahren & $\begin{array}{l}\text { Ggf. rasche Einschätzung, welche Be- } \\
\text { handlungsform prinzipiell möglich }\end{array}$ & $\begin{array}{l}\text { Eingeschränkte körperliche Untersuchung } \\
\text { Fehlende Zusatzuntersuchungen } \\
\text { Teils fehlende Möglichkeit, Gerät zu prüfen } \\
\text { Justiziabilität (Prüfpflicht)? }\end{array}$ \\
\hline
\end{tabular}

sondere hinsichtlich Qualität, ist jedoch stets Rechnung zu tragen.

\section{Korrespondenzadresse}

\section{Dr. Randi von Wrede}

Klinik und Poliklinik für Epileptologie,

Universitätsklinikum Bonn

Venusberg Campus 1, 53127 Bonn,

Deutschland

randi.von.wrede@ukbonn.de

\section{Einhaltung ethischer Richtlinien}

Interessenkonflikt. R. von Wrede hat in den letzten 5 Jahren Honorare für Vorträge, als Berater in oder Reiskostenunterstützung von Arvelle, Cerbomed, Desitin, GW-Pharma, Eisai, UCB Pharma erhalten. R. Surges hat in den letzten 5 Jahren Honorare für Vorträge oder als Berater von Arvelle, Angelini Bial, Desitin, Eisai, LivaNova, Novartis, UCB Pharma und UNEEG erhalten
Für diesen Beitrag wurden von den Autoren keine Studien an Menschen oder Tieren durchgeführt. Für die aufgeführten Studien gelten die jeweils dort angegebenen ethischen Richtlinien.

\section{Literatur}

1. Bahrani K, Singh MB, Bhatia R et al (2017) Telephonic review for outpatients with epilepsy-A prospective randomized, parallel group study. Sei- 
zure 53:55-61. https://doi.org/10.1016/j.seizure. 2017.11.003

2. Banks J, Corrigan D, Grogan R et al (2021) LoVE in a time of CoVID: Clinician and patient experience using telemedicine for chronic epilepsy management. Epilepsy Behav 115:107675.https:// doi.org/10.1016/j.yebeh.2020.107675

3. Beck CA, Beran DB, Biglan KM et al (2017) National randomized controlled trial of virtual house calls for Parkinson disease. Neurology 89:1152-1161. https://doi.org/10.1212/WNL. 0000000000004357

4. Bloem BR, Dorsey ER, Okun MS (2020) The coronavirus disease 2019 crisis as catalyst for telemedicine for chronic neurological disorders. JAMA Neurol 77:927-928. https://doi.org/10. 1001/jamaneurol.2020.1452

5. Casares M, Wombles C, Skinner HJ et al (2020) Telehealth perceptions in patients with epilepsy and providers during the COVID-19 pandemic. Epilepsy Behav 112:107394. https://doi.org/10. 1016/j.yebeh.2020.107394

6. Datta P, Barrett W, Bentzinger M et al (2021) Ambulatory care for epilepsy via telemedicine during the COVID-19 pandemic. Epilepsy Behav 116:107740. https://doi.org/10.1016/j.yebeh 2020.107740

7. Fesler JR, Stanton S, Merner Ket al (2020) Bridging the gap in epilepsy care: a single-center experience of 3700 outpatient tele-epilepsy visits. Epilepsia 61:e95-e100.https://doi.org/10.1111/epi.16619

8. Fonseca E, Quintana M, Lallana S et al (2020) Epilepsy in time of COVID-19: a survey-based study. Acta Neurol Scand 142:545-554. https://doi.org/ 10.1111/ane.13335

9. Ha JF, Longnecker N (2010) Doctor-patient communication: a review. Ochsner J 10:38-43

10. Hatcher-Martin JM, Adams JL, Anderson ER et al (2020) Telemedicine in neurology. Neurology 94:30-338. https://doi.org/10.1212/WNL. 0000000000008708

11. (2021) https://www.bundesaerztekammer.de/ fileadmin/user upload/downloads/pdf-Ordner/ Recht/HinweiseErlaeuterungenFernbehandlung. pdf.Zugegriffen: 25.06.2021

12. (2021) https://de.wikipedia.org/wiki/Telemedizin. Zugegriffen: 25.06.2021
13. https://www.kbv.de/media/sp/BMV-Aerzte.pdf. Zugegriffen: 25.06 .2021

14. (2021) https://www.kbv.de/media/sp/Liste zertifizierte-Videodienstanbieter.pdf. Zugegriffen: 25.06 .2021

15. (2021)https://www.kbv.de/media/sp/Videosprech stunde_uebersicht_Verguetung.pdf. Zugegriffen:25.06.2021

16. https://www.zaoerv.de/01_1929/1_1929_2_b_ 536_2_541.pdf.Zugegriffen:25.06.2021

17. Kuchenbuch M, D'Onofrio G, Wirrell E et al (2020) An accelerated shift in the use of remote systems in epilepsy due to the COVID-19 pandemic. Epilepsy Behav 112:107376. https://doi.org/10. 1016/j.yebeh.2020.107376

18. Kuroda N (2020) Decision making on telemedicine for patients with epilepsy during the Coronavirus disease 2019 (COVID-19) crisis. Front Neurol 11:722. https://doi.org/10.3389/fneur.2020.00722

19. Lurie N, Carr BG (2018) The role of telehealth in the medical response to disasters. JAMA Intern Med 178:745-746. https://doi.org/10.1001/ jamainternmed.2018.1314

20. Mostacci B, Licchetta L, Cacciavillani C et al (2020) The impact of the COVID-19 pandemic on people with epilepsy. An Italian survey and a global perspective. Front Neurol 11:613719. https://doi. org/10.3389/fneur.2020.613719

21. PattersonV(2019) Managing epilepsyby telemedicine in resource-poor settings. Front Public Health 7:1-6. https://doi.org/10.3389/fpubh.2019.00321

22. Peine A, Paffenholz P, Martin L et al (2020) Telemedicine in Germany during the COVID-19 pandemic: multi-professional national survey. J Med Internet Res 22:e19745. https://doi.org/10 2196/19745

23. Rasmusson KA, Hartshorn JC (2005) A comparison of epilepsy patients in a traditional ambulatory clinic and a telemedicine clinic. Epilepsia 46:767-770. https://doi.org/10.1111/j.15281167.2005.44804.x

24. Sattar S, Kuperman R (2020) Telehealth in pediatric epilepsy care: a rapid transition during the COVID19 pandemic. Epilepsy Behav 111:107282. https:// doi.org/10.1016/j.yebeh.2020.107282

25. Wechsler LR, Tsao JW, Levine SR et al (2013) Teleneurology applications: report of the tele- medicine work group of the American Academy of Neurology. Neurology 80:670-676. https://doi. org/10.1212/WNL.0b013e3182823361

26. Willems LM, Balcik Y, Noda AH et al (2020) SARSCoV-2-related rapid reorganization of an epilepsy outpatient clinic from personal appointments to telemedicine services: a German single-center experience. Epilepsy Behav 112:107483. https:// doi.org/10.1016/j.yebeh.2020.107483

27. von Wrede R, Moskau-Hartmann S, Baumgartner T et al (2020) Counseling of people with epilepsy via telemedicine: experiences at a German tertiary epilepsy center during the COVID-19 pandemic. Epilepsy Behav 112:107298

Wir sagen Danke! Springer Medizin spendet im Jahr 2021 10.000 EUR an „Ärzte ohne Grenzen" für Gutachten zu eingereichten Beiträgen. 